Review

\title{
Nonlinear control of flap-type wave energy converter with a non-ideal power take-off system ${ }^{\text {is }}$
}

\author{
G. Bacelli ${ }^{1, *}$, R. Genest, J.V. Ringwood \\ Centre for Ocean Energy Research, National University of Ireland Maynooth, Maynooth, County Kildare, Ireland
}

\section{A R T I C L E I N F O}

\section{Article history:}

Received 8 May 2015

Accepted 4 August 2015

Available online 16 October 2015

\section{Keywords:}

Wave energy devices

Nonlinear modelling

\begin{abstract}
A B S T R A C T
Wave energy converters (WECs) require active control to maximise energy capture over a wide range of sea conditions, which is generally achieved by making the device resonate. The exaggerated device motion arising at resonance, however, may result in nonlinear effects that are ignored by the linear models that are typically employed. In particular, nonlinear viscous forces are significant for particular device types, such as hinged flaps, which we take as a case study in this paper. The paper develops a general nonlinear WEC control methodology based on pseudospectral methods. The continuous time energy maximisation problem is fully discretised (both state and control), and the optimal solution is obtained by solving the resulting finite dimensional optimisation problem. By way of example, the nonlinear viscous damping for a hinged flap WEC is incorporated into the control model which also considers non-ideal power take-off efficiency. It is shown that the ratio of energy captured to energy dissipated is significantly increased with the nonlinear controller, compared to the linear case.
\end{abstract}

(c) 2015 International Federation of Automatic Control. Published by Elsevier Ltd. All rights reserved.

\section{Introduction}

Wave energy conversion is the process of transforming energy carried by water waves in the sea into a usable form of energy, e.g. electricity. Devices designed to fulfil this task are known as Wave Energy Converters (WECs), and this paper concerns the control of a particular type of device, where the objective of the control system is to maximise the amount of energy absorbed. The device considered in this paper is a bottom-hinged vertical plate (Fig. 1) which exploits the same conversion principle as the Oyster WEC being developed by Aquamarine Power Ltd. (Folley, Whittaker, \& van't Hoff, 2007). The force exerted by the incident waves (excitation force) induces a pitching motion on the plate. Part of the mechanical work done by the excitation force is converted into a usable form of energy by means of the Power Take Off (PTO), a component of the WEC capable of doing mechanical work on the oscillating plate by exerting a force, which is the control variable.

Most studies, academic and commercial, focus on the use of linear models; their appeal is mainly due to the possibility of developing

\footnotetext{
A shorter version of this paper was presented at the 19th IFAC World Congress, Cape Town, South Africa, August 24-29, 2014

* Corresponding author.

E-mail addresses: gbacell@sandia.gov (G. Bacelli), romain.genest@eeng.nuim.ie (R. Genest), john.ringwood@eeng.nuim.ie (J.V. Ringwood).

1 This work was completed while G. Bacelli was affiliated with the Centre for Ocean Energy Research at Maynooth University. G.Bacelli is currently with Sandia National Laboratories, Albuquerque, NM, USA.
}

analytical solutions for the control problems and analysis of performance (Falnes, 2002). A variety of sources introduce nonlinearities in the model of WECs, from the PTO (Bacelli, Gilloteaux, \& Ringwood, 2008; Engja \& Hals, 2007) to the fluid-body interactions. While it is often reasonable to assume a linear approximation for the radiation (Gilloteaux, 2007), some studies have shown the wide disparity between linear and nonlinear models of excitation forces (Merigaud, Gilloteaux, \& Ringwood, 2012), viscous forces (Folley et al., 2007) and hydrostatic restoring forces (Zurkinden \& Kramer, 2012). This paper focusses on viscous drag applied to a hinged flap WEC as an example to illustrate the application of the pseudospectral methods for the nonlinear control of wave energy converters. In addition, nonlinearities coming from non-ideal PTO losses are studied and modelled via an efficiency curve. An investigation is carried out on a generic hinged flap device to illustrate how PTO losses can be taken into account during the optimal trajectory generation using pseudospectral methods. However, the nonlinear control framework is general and can be applied to other nonlinearities and WEC device types. An initial exposure of the nonlinear control of a flap-type WEC using pseudo-spectral methods was presented in (Bacelli \& Ringwood, 2014), though a non-ideal PTO was not considered.

The control problem is an optimal control problem because the objective is to find the control (PTO force) which maximises the amount of absorbed energy. In this paper, the solution to the nonlinear optimal control of a WEC is obtained by means of pseudospectral methods, which are a subset of the class of techniques used for the discretisation of integral and partial differential equations, known 


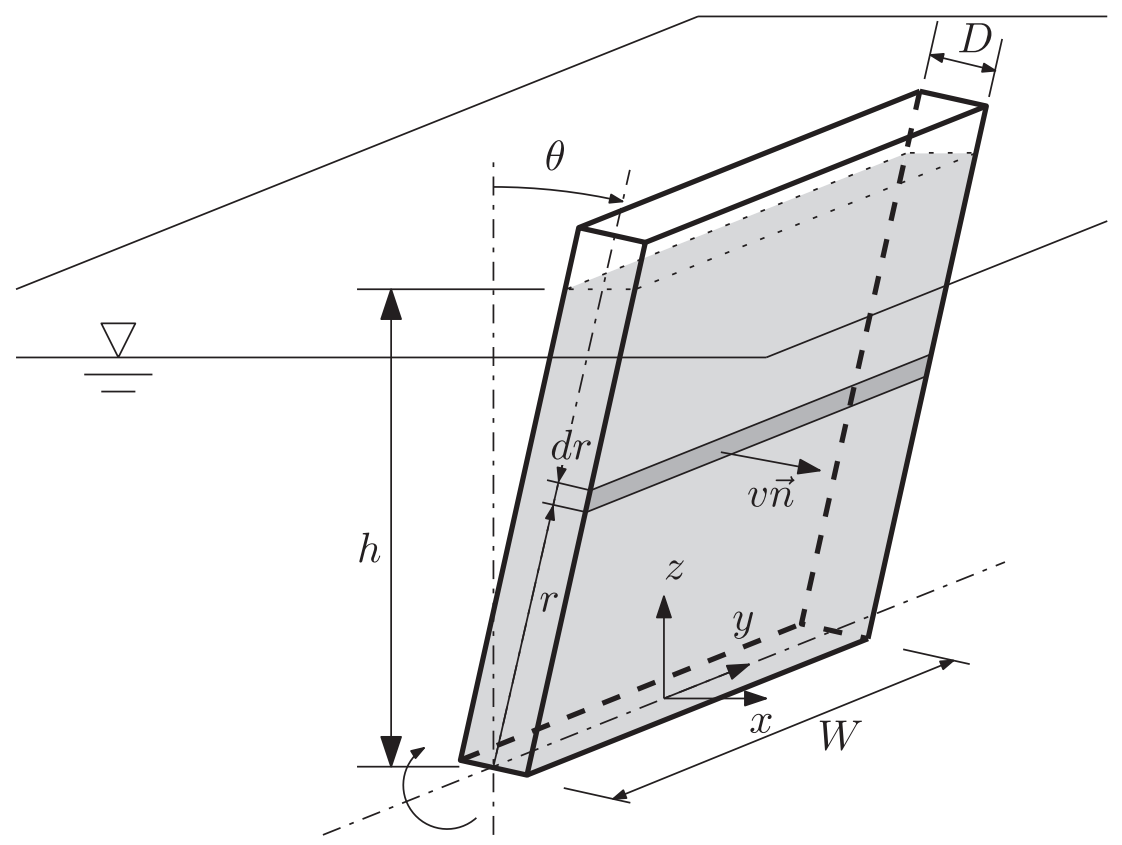

Fig. 1. Flap-type wave energy converter. The shaded area indicates the submerged region.

as mean weighted residuals (Canuto, Hussaini, Quarteroni, \& Zang, 2006; Fornberg, 1996). The first applications of pseudospectral optimal control were presented more than 15 years ago (Elnagar, Kazemi, \& Razzaghi, 1995; Vlassenbroeck \& Van Dooren, 1988); however, only in recent years has it received increasing attention (Garg et al., 2010; Ross \& Karpenko, 2012) and found application, mostly in flight control.

Adopting an optimal numerical framework gives the opportunity to deal with the full complexity of the device model (possibly including nonlinear terms and non-ideal PTO), device constraints and optimising the device for the a panchromatic wave spectrum, where multiple frequencies are simultaneously present.

Previous approaches to nonlinear control of WECs include the application of Pontryagin's maximum principle to the continuous time optimal control problem (Babarit \& Clément, 2006; Nielsen, Zhou, Kramer, Basu, \& Zhang, 2013) and its discretisation (Richter, Magana, Sawodny, \& Brekken, 2013; Tom \& Yeung, 2013). However, discretisation using pseudospectral methods generally gives a faster convergence rate (Benson, 2005), which results in a smaller nonlinear program and reduced computing time, thus suitable for real-time applications. Additionally, discretisation by means of the pseudospectral method presented in this paper allows the convolution integral that models the radiation force to be simplified analytically, instead of the classical approach of using system identification to build a state space model (Tom \& Yeung, 2013) or being completely neglected (Richter et al., 2013).

Since more and more new devices and prototypes are being tested in wave tanks or under real sea conditions, WECs dealing with nonideal PTO systems becomes a new issue and a contemporary technological challenge. Solutions have been proposed by Hansen, Pedersen, and Andersen (2014) for hydraulic PTO systems, replacing on/off valves by bidirectional check valves in order to reduce switching losses. Other recent studies, such as Tedeschi, Carraro, Molinas, and Mattavelli (2011), Kovaltchouk et al. (2013) and Genest, Bonnefoy, Clément, and Babarit (2014), evaluate the impact of power take-off losses on the absorbed power of generic wave energy converters using efficiency curves or a constant efficiency rate for electrical or hydraulic PTO systems. Such studies illustrate how essential it is to take into account PTO losses in the control strategy since, even with a high efficiency PTO, the absorbed grid power significantly drops in comparison to the ideal case.
The remainder of the paper is organised as follows: the dynamical model of the flap-type WEC is described in Section 2, and a brief overview of pseudospectral optimal control is provided in Section. 3, while Section 4 shows a case study for the flap-type device. Inclusion of a nonideal PTO in the pseudospectral optimal control is introduced in Section 5 and simulation results are illustrated and discussed in Section 6, with conclusions drawn in Section. 7.

\section{WEC model}

\subsection{Dynamical model}

The device considered in this paper is depicted in Fig. 1. It is a flap-type WEC hinged on the $y$-axis at a depth $h=15 \mathrm{~m}$, with a width $W=30 \mathrm{~m}$, thickness $D=1 \mathrm{~m}$ and a uniform density $\rho_{b}=250 \mathrm{~kg} / \mathrm{m}^{3}$.

The equation of motion is derived from Euler's second law, which says that the rate of change of the angular momentum is equal to the sum of the external moments of force about the axis $y$ :

$I_{y} \ddot{\theta}=\gamma_{w}(t)+\gamma_{p}(t)$.

$I_{y}$ is the moment of inertia of the body with respect to the axis $y, \gamma_{p}$ is the torque applied by the PTO, and $\gamma_{w}$ is the resultant of the moments due to the interaction between water and the oscillating body, which is composed of four terms, as described by Folley et al. (2007):

$\gamma_{w}(t)=\gamma_{h}(t)+\gamma_{d}(t)+\gamma_{r}(t)+\gamma_{e}(t)$.

The hydrostatic restoring moment $\gamma_{h}$ is assumed to be linearly proportional to the pitch angle $\left(\gamma_{h}=S_{h} \theta\right)$, where $S_{h}$ is the hydrostatic restoring coefficient. The excitation torque $\gamma_{e}$ is due to the effect of the incident waves, and is calculated as $\gamma_{e}(t)=h_{e} * \zeta$, where $\zeta$ is the wave elevation and $*$ denotes the convolution operator, defined by

$f * g=\int_{-\infty}^{-\infty} f(t-\tau) g(\tau) d \tau$.

The radiation torque $\gamma_{r}$ is due to the motion of the body which causes waves to be radiated away, and depends on the velocity and acceleration of the oscillating body as (Cummins, 1962):

$\gamma_{r}=-I_{\infty} \ddot{\theta}-h_{r} * \dot{\theta}$

The functions $h_{e}$ and $h_{r}$ are the impulse responses of the excitation and radiation respectively, while $I_{\infty}$ is the asymptotic value of the 
added inertia for "infinite frequency" (Falnes, 2002). The values of $h_{e}$, $h_{r}$ and $I_{\infty}$ are calculated using the boundary element software WAMIT (2013).

The nonlinear part of the dynamic model is due to the moment of the drag force $\left(f_{d}\right)$, which is generally modelled as proportional to the square of the fluid velocity normal to the surface of the body (Journé \& Massie, 2001):

$f_{d}=-(1 / 2) \rho C_{d} A v|v|$,

where $\rho$ is the density of the water, $C_{d}$ is the drag coefficient, $A$ is the area normal to the direction of the relative fluid flow and $v$ is the velocity normal to the surface (Fig. 1). When the body is in the vertical position $(\theta=0)$, for small oscillations, the normal velocity on the vertical surface is related to the angular velocity as $v \approx r \dot{\theta}$, where $r$ is the vertical distance between the hinge and the point of the surface where the velocity is considered. The contribution to the drag force of the infinitesimal surface at a distance $r$ from the hinge, of width $W$ and height $d r$ (Fig. 1$)$ is $d f_{d} \approx-(1 / 2) \rho C_{d} W r^{2} \dot{\theta}|\dot{\theta}| d r$. The infinitesimal moment of the drag force applied with respect to the axis $y$ is $d \gamma_{v}=r d f_{d}$; by integrating $d \gamma_{v}$ from 0 to $h$, the total moment of the drag force applied to the hinge is:

$\gamma_{v}=-\frac{1}{2} \int_{0}^{h} \rho C_{d} W r^{3} \dot{\theta}|\dot{\theta}| d r=-B_{v_{2}} \dot{\theta}|\dot{\theta}|$

where $B_{v_{2}}=(1 / 8) \rho C_{d} W h^{4}$. According to Blevins (1992), the drag coefficient of a plate orthogonal to the direction of the flow is $C_{d}=1.9$ (Blevins, 1992). The presence of viscous drag for a surging plate is further confirmed in Bhinder, Babarit, Gentaz, and Ferrant (2015), where a surging plate is examined and a viscous drag model determined using computational fluid dynamics (CFD) techniques. A drag coefficient of $C_{d}=1.8$ is shown to validate well with the CFD simulations. In addition, the study in Chen, Fang, Li, Huang, and Chung (2009) suggests a drag coefficient in the range $6<C_{d}<7$ for a sharp-edged plate, corresponding to Kergeulen-Carpenter $(K C)$ values of $1 \leq K C \leq$ 10. Finally, it is important to realise that the relative device/fluid velocity (with resulting greater nonlinear viscous drag) is greater with the application of control forces than for studies where wave excitation only is considered, as documented in Ringwood, Davidson, and Giorgi (2015). By way of example, the flap tip velocity in the current case will approach $2.6 \mathrm{~m} / \mathrm{s}$ by virtue of the control force applied.

The resulting equation of motion is

$I_{t} \ddot{\theta}=-B_{v_{1}} \dot{\theta}-B_{v_{2}} \dot{\theta}|\dot{\theta}|-h_{r} * \dot{\theta}-S_{h} \theta+\gamma_{p}+\gamma_{e}$.

where $I_{t}=\left(I_{y}+I_{\infty}\right)$, and $B_{v_{1}}$ is the coefficient of a linear dissipative term, which models additional losses occurring at small velocities, when the effect of the quadratic term is negligible (Journe \& Massie, 2001).

\subsection{Non-ideal power take-off model}

PTO losses constitute a crucial technological aspect that must be taken into consideration in the wave energy control design process. Inclusion of a non-ideal PTO in the formulation of the control is, in this way, essential. Different levels of complexity can be used to describe the PTO behaviour. A complete technical descriptive approach could be employed, leading to a more realistic model, but resulting in a significant increase in computation time, obviously prejudicial if used in a control design loop. A simpler approach is to exclusively model energy PTO losses by means of efficiency curves or a constant efficiency rate. Efficiency curves can be a function of various parameters such as, for example, load factor, the duration and frequency of use, or temperature. A generic efficiency curve is used in this study, which is a function of the load factor only. Our intention, in the present work, is not to provide an exact characteristic curve modelling any specific PTO technology, but rather to illustrate how pseudospectral optimal control can handle nonlinearity originating

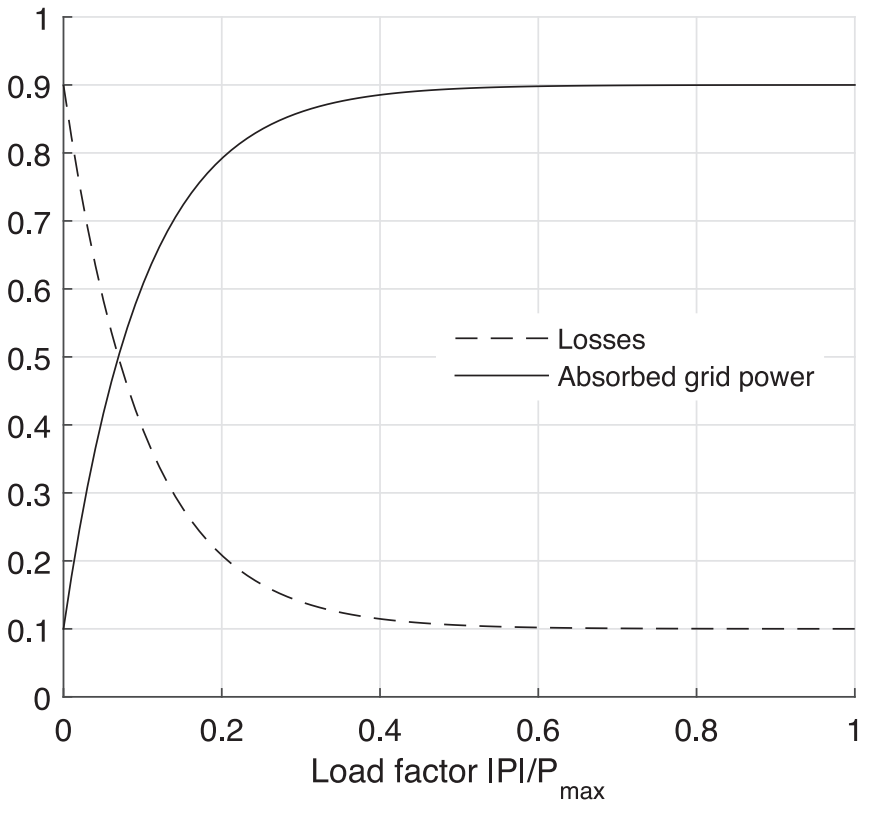

Fig. 2. Losses and absorbed grid power function of the load factor.

from PTO energy losses, as well as nonlinear hydrodynamics effects. A more realistic efficiency curve, based on a particular PTO device, would constitute a further step in the control design, but is not the focus of the present paper. The expression of the PTO losses, $L$, used here is defined by Eq. (7) as a function of the load factor, $r$, expressed as a percentage of full load.

$L(r)=\left(L_{i n i}-L_{\min }\right) e^{-\tau r}+L_{\text {min }}, \forall r \in[0,1]$

where $L$ corresponds to the fraction of lost power, i.e. the actual lost power is determined by multiplying $L$ by the absolute value of the absorbed power coming from the PTO, $P_{\text {losses }}=L(r)|P| . r=|P| / P_{\max }$ is the load factor and takes values between 0 , where no load is required, and 1 , where the maximum load is needed to control the WEC. An example of an efficiency curve is shown in Fig. 2 with the following parameters : $L_{i n i}=0.9, L_{\min }=0.1, \tau=10$. The actual power absorbed and delivered to the grid, $P_{\text {grid }}$, is equal to the absorbed power from the PTO reduced by the PTO losses and is defined as

$P_{\text {grid }}(P)=P-L(r)|P|, \forall P \in\left[-P_{\max }, P_{\max }\right]$

where $P$ is the power absorbed by the PTO, i.e. $P=-\gamma_{p} \dot{\theta}$, with the control torque $\gamma_{p}$ and the velocity of the device $\dot{\theta}$. It can be noticed that the cost function becomes clearly nonlinear and implicitly entails a nonlinear constraint on the absorbed PTO power, i.e. a PTO power limitation.

\section{Pseudospectral optimal control}

Pseudospectral optimal control is a method for the direct transcription of an optimal control problem (Ross \& Karpenko, 2012), which means that both control and state variables are discretised, and the original control problem is approximated by a nonlinear program.

Consider, for example, the optimal control problem: determine the control, $\boldsymbol{u}(t) \in \mathbb{R}^{m}$, that minimises, or maximises, the cost functional in the Lagrange form (Stengel, 1986):

$J=\int_{0}^{T} g(\boldsymbol{x}, \boldsymbol{u}, t) d t, \quad g: \mathbb{R}^{n} \times \mathbb{R}^{m} \times \mathbb{R} \rightarrow \mathbb{R}$

subject to the dynamic constraint

$\dot{\boldsymbol{x}}=\boldsymbol{f}(\boldsymbol{x}, \boldsymbol{u}, t) \quad t \in[0, T]$,

where $\boldsymbol{x}(t) \in \mathbb{R}^{n}$ and $\boldsymbol{f}: \mathbb{R}^{n} \times \mathbb{R}^{m} \times \mathbb{R} \rightarrow \mathbb{R}^{n}$. 
The first step is to approximate the state and control variables by considering, for the $i$-th components, the following expansion:

$x_{i}(t) \approx x_{i}^{N}(t):=\sum_{k=1}^{N} \hat{x}_{i k} \phi_{k}(t)=\Phi(t) \hat{\boldsymbol{x}}_{i}$

$u_{i}(t) \approx u_{i}^{N}(t):=\sum_{k=1}^{N} \hat{u}_{i k} \phi_{k}(t)=\Phi(t) \hat{\boldsymbol{u}}_{i}$

where

$\hat{\boldsymbol{x}}_{i}=\left[\hat{x}_{i 1}, \hat{x}_{i 2}, \ldots, \hat{x}_{i N}\right]^{T}$,

$\hat{\boldsymbol{u}}_{i}=\left[\hat{u}_{i 1}, \hat{u}_{i 2}, \ldots, \hat{u}_{i N}\right]^{T}$,

and

$\Phi(t)=\left[\phi_{1}(t), \phi_{2}(t), \ldots, \phi_{N}(t)\right]$

form a basis for an $\mathrm{N}$-dimensional vector space, on which the state and control variables are approximated. It is also convenient to define the vectors $\boldsymbol{X} \in \mathbb{R}^{N n}$ and $\boldsymbol{U} \in \mathbb{R}^{N m}$ :

$\boldsymbol{X}=\left[\hat{\boldsymbol{x}}_{1}^{T}, \ldots, \hat{\boldsymbol{x}}_{n}^{T}\right]^{T} \quad \boldsymbol{U}=\left[\hat{\boldsymbol{u}}_{1}^{T}, \ldots, \hat{\boldsymbol{u}}_{n}^{T}\right]^{T}$.

As the result of the approximations, the cost functional (9) depends only on the $N(n+m)$ coefficients in $\boldsymbol{X}$ and $\boldsymbol{U}$, thus the optimisation problem is finite dimensional.

To illustrate the effect of the approximation on the dynamic equation, the derivative of the approximated state variable is considered first, that is:

$\dot{x}_{i}^{N}=\sum_{k=1}^{N} \hat{x}_{i k} \dot{\phi}_{k}(t)=\dot{\Phi}(t) \hat{\boldsymbol{x}}_{i}$

By substituting (11), (12) and (13) into (10), the approximated dynamic equation in the residual form is then

$r_{i}(t)=\dot{x}_{i}^{N}(t)-f_{i}\left(\boldsymbol{x}^{N}(t), \boldsymbol{u}^{N}(t), t\right), \quad i=1, \ldots, n$

where $\boldsymbol{x}^{N}$ and $\boldsymbol{u}^{N}$ are the vectors of the approximated state variables and control variables respectively, the elements of which are $x_{i}^{N}$ defined in (11) and $u_{i}^{N}$ defined in (12). The coefficients $\hat{\boldsymbol{x}}_{i}$ and $\hat{\boldsymbol{u}}_{j}$ for which the $n$ residuals (14) are minimised are calculated by using the pseudospectral method (Elnagar et al., 1995), also known as the collocation method. The method consists of collocating the system dynamics at a number of time points $t_{k}$, called nodes, meaning that the coefficients $\hat{\boldsymbol{x}}_{i}$ and $\hat{\boldsymbol{u}}_{j}$ are such that the dynamic equation is satisfied at a number of points $t_{k}$, or equivalently, the residuals $r_{i}$ are zero at the $N_{c}$ nodes:

$r_{i}\left(t_{j}\right)=\dot{\Phi}\left(t_{j}\right) \hat{\boldsymbol{x}}_{i}-f_{i}\left(\boldsymbol{X}, \boldsymbol{U}, t_{j}\right)=0$

which is a system of $n \times N_{c}$ equations because $j=1, \ldots, N_{c}$ and $i=$ $1, \ldots, n$.

The functional $J$ in (9) is also approximated by an appropriate quadrature formula with weights $w_{j}$, as

$J^{N}=\int_{0}^{T} g(\boldsymbol{X}, \boldsymbol{U}, t) d t \approx \sum_{j=0}^{N_{c}} g\left(\boldsymbol{X}, \boldsymbol{U}, t_{j}\right) w_{j}$,

and the optimal control problem defined by the cost functional (9) and the dynamic constraint (10) is transformed into the finite dimensional optimisation problem: find $\boldsymbol{U}$ and $\boldsymbol{X}$ to maximise (or minimise) (16) subject to the constraints (15).

The collocation of the dynamic equation and of the cost functional, that is the choice of the nodes $t_{j}$, depends on a number of factors, including the expansions (11) and (12) (Garg et al., 2010; Ross \& Karpenko, 2012).

\section{Optimal WEC control}

The optimal control problem that we are aiming to solve is the maximisation of the absorbed energy, which is equivalent to maximising the amount of work done by the PTO moment

$J=\int_{0}^{T} \gamma_{p}(t) \dot{\theta}(t) d t$

subject to the constraint given by the dynamic model in (6). The first step is to choose the expansion for the state and control and, given the oscillatory nature of the problem, a zero-mean trigonometric polynomial (truncated Fourier series) is a sensible choice, thus:

$x_{i}(t) \approx \sum_{k=1}^{N / 2} x_{i k}^{c} \cos \left(k \omega_{0} t\right)+x_{i k}^{s} \sin \left(k \omega_{0} t\right)=\Phi(t) \hat{\boldsymbol{x}}_{i}$

$u(t) \approx \sum_{k=1}^{N / 2} u_{k}^{c} \cos \left(k \omega_{0} t\right)+u_{k}^{s} \sin \left(k \omega_{0} t\right)=\Phi(t) \hat{\boldsymbol{u}}$

where

$\hat{\boldsymbol{x}}_{i}=\left[x_{i 1}^{c}, x_{i 1}^{S}, \ldots, x_{i \frac{N}{2}}^{c}+x_{i \frac{N}{2}}^{S}\right]^{T}$

$\hat{\boldsymbol{u}}=\left[u_{1}^{c}, u_{1}^{s}, \ldots, u_{\frac{N}{2}}^{c}+u_{\frac{N}{2}}^{s}\right]^{T}$

$\Phi(t)=\left[\cos \left(\omega_{0} t\right), \sin \left(\omega_{0} t\right), \ldots, \cos \left(\frac{N}{2} \omega_{0} t\right), \sin \left(\frac{N}{2} \omega_{0} t\right)\right]$

where the fundamental frequency is $\omega_{0}=2 \pi / T$.

By substituting the state (18) and control (19) expansions into the cost function (17), the approximated absorbed energy is

$J^{N}=\int_{0}^{T} \hat{\boldsymbol{u}}^{T} \Phi^{T}(t) \Phi(t) \hat{\boldsymbol{x}}_{2} d t=\frac{T}{2} \hat{\boldsymbol{u}}^{T} \hat{\boldsymbol{x}}_{2}$,

because of the orthogonality of the basis $\Phi$, that is $\left\langle\phi_{i}, \phi_{j}\right\rangle=\delta_{i j} T / 2$, where $\delta_{i j}$ is the Kronecker delta.

The derivative of the state variables in (13), given the approximation of the state in (18), becomes

$\dot{x}_{i}^{N}=\dot{\Phi}(t) \hat{\boldsymbol{x}}_{i}=\Phi(t) \mathrm{D}_{\phi} \hat{\boldsymbol{x}}_{i}$

where $\mathrm{D}_{\phi} \in \mathbb{R}^{N \times N}$ is a block diagonal matrix, with the $k$-th block defined as

$\mathrm{D}_{\phi}^{k}=\left[\begin{array}{cc}0 & k \omega_{0} \\ -k \omega_{0} & 0\end{array}\right]$

The state vector is composed of the angular position and velocity, that is, $\boldsymbol{x}=\left[x_{1}, x_{2}\right]^{T}=[\theta, \dot{\theta}]^{T}$, and the control input is the PTO moment $\left(u=\gamma_{p}\right)$, thus $n=2$ and $m=1$. Consequently, the dynamic equation (6) can be transformed into the system of equations:

$\dot{x}_{1}=x_{2}$

$I_{t} \dot{x}_{2}=-B_{v_{1}} x_{2}-B_{v_{2}} x_{2}\left|x_{2}\right|-h_{r} * x_{2}-S_{h} x_{1}+u-\gamma_{e}$

By applying the approximations (18) and (21) to the first state equation (22), the result is

$\Phi(t) \mathrm{D}_{\phi} \hat{\boldsymbol{x}}_{1}-\Phi(t) \hat{\boldsymbol{x}}_{2}=0 \quad \Leftrightarrow \quad \mathrm{D}_{\phi} \hat{\boldsymbol{x}}_{1}-\hat{\boldsymbol{x}}_{2}=0$.

because the elements of $\Phi$ form a basis.

The residuals of the second state equation (23), collocated at the nodes $t_{j}$ are

$r_{j}=\mathrm{I}_{t} \Phi_{j} \mathrm{D}_{\phi} \hat{\boldsymbol{x}}_{2}+B_{v_{1}} \Phi_{j} \hat{\boldsymbol{x}}_{2}+B_{v_{2}} \Phi_{j} \hat{\boldsymbol{x}}_{2}\left|\Phi_{j} \hat{\boldsymbol{x}}_{2}\right|+S_{h} \Phi_{j} \hat{\boldsymbol{x}}_{1}$

$+\left(h_{r} * \Phi\right)_{t_{j}} \hat{\boldsymbol{x}}_{2}-\Phi_{j} \hat{\boldsymbol{u}}-\gamma_{e}\left(t_{j}\right)$.

where $\Phi_{j}=\Phi\left(t_{j}\right)$. The convolution term can be simplified by substituting the approximation (18) into the convolution integral; after 

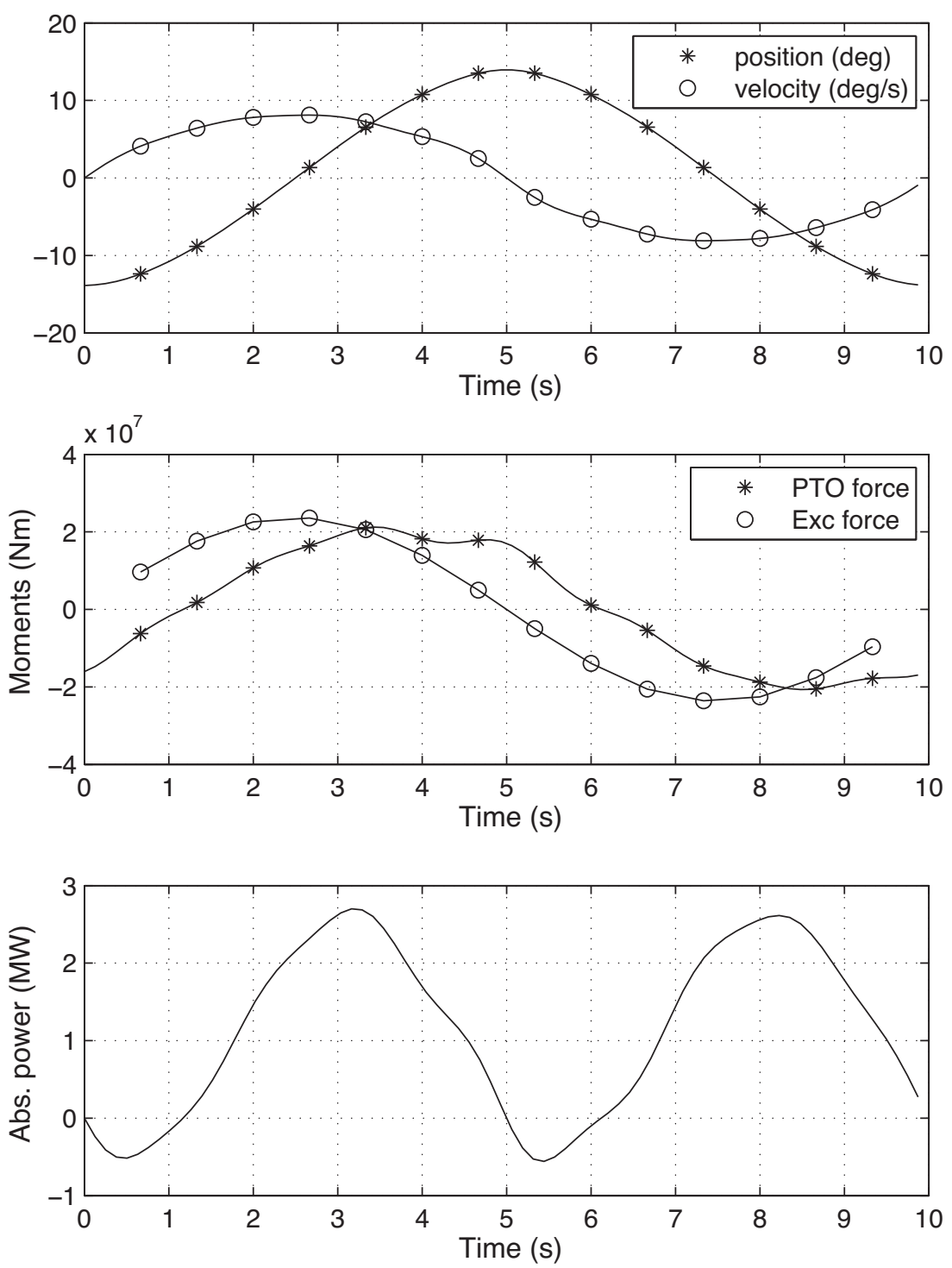

Fig. 3. Motion, forces and absorbed power for $T=10 \mathrm{~s}, \zeta_{a}=2 \mathrm{~m}$ and $N=14$.

some basic derivations involving trigonometric identities and the definition of the sine and cosine transforms, which we omit for brevity, the result is

$$
\begin{aligned}
\left(h_{r} * \Phi\right)_{t_{j}} \hat{\boldsymbol{x}}_{2} & =\int_{-\infty}^{+\infty} h_{r}\left(t_{j}-\tau\right) x_{2}^{N}(\tau) d \tau \\
& =\Phi_{j}\left(\mathrm{G}-I_{\infty} \mathrm{D}_{\Phi}\right) \hat{\boldsymbol{x}}_{2},
\end{aligned}
$$

where the matrix $G \in \mathbb{R}^{N \times N}$ is block diagonal, and the $k$-th block is

$\mathrm{G}_{k}=\left[\begin{array}{cc}B\left(k \omega_{0}\right) & k \omega_{0} A\left(k \omega_{0}\right) \\ -k \omega_{0} A\left(k \omega_{0}\right) & B\left(k \omega_{0}\right)\end{array}\right]$.

The frequency domain coefficients $A$ and $B$ are related to the impulse responses by means of the Cummins relations (Cummins, 1962), and they are provided directly by WAMIT (2013).

Substituting (27) into (25), the residuals simplify to

$$
\begin{aligned}
r_{j}= & I_{y} \Phi_{j} \mathrm{D}_{\phi} \hat{\boldsymbol{x}}_{2}+B_{v_{1}} \Phi_{j} \hat{\boldsymbol{x}}_{2}+B_{v_{2}} \Phi_{k} \hat{\boldsymbol{x}}_{2}\left|\Phi_{j} \hat{\boldsymbol{x}}_{2}\right|+\Phi_{j} \mathrm{G} \hat{\boldsymbol{x}}_{2}+S_{h} \Phi_{j} \hat{\boldsymbol{x}}_{1} \\
& -\Phi_{j} \hat{\boldsymbol{u}}-\gamma_{e}\left(t_{j}\right)=0 .
\end{aligned}
$$

where the nodes $t_{j}$ are uniformly spaced between 0 and $T-\Delta t$ :

$t_{j}=j \Delta t, \quad$ with $\Delta t=T /(N+1)$ and $j=0, \ldots, N$.
The vectors $\boldsymbol{U}$ and $\boldsymbol{X}$, that give the optimal profile for the PTO moment and the motion of the flap, respectively, are the solutions of the nonlinear program which maximises the absorbed energy (20), subject to the $2 \mathrm{~N}$ equality constraints due to the dynamic equations (24) and (28).

\section{Non-ideal PTO}

Assuming a non-ideal PTO, the expression of the cost function must be based on the actual grid power, instead of the power directly absorbed by the PTO, as

$J^{N}=\int_{0}^{T} P_{\text {grid }}(t) d t$

Replacing $P_{\text {grid }}$ by its expression from Eq. (8), we obtain,

$J^{N}=\int_{0}^{T}(P(t)-L(r(t))|P(t)|) d t$

with $P=\hat{\mathbf{u}}^{T} \Phi^{T} \Phi \hat{\mathbf{x}}_{2}$ and $r=|P| / P_{\max }$. The cost function $J^{N}$ cannot be simplified as was previously done in Eq. (20) and has to be determined using a quadrature rule to perform the numerical integration 

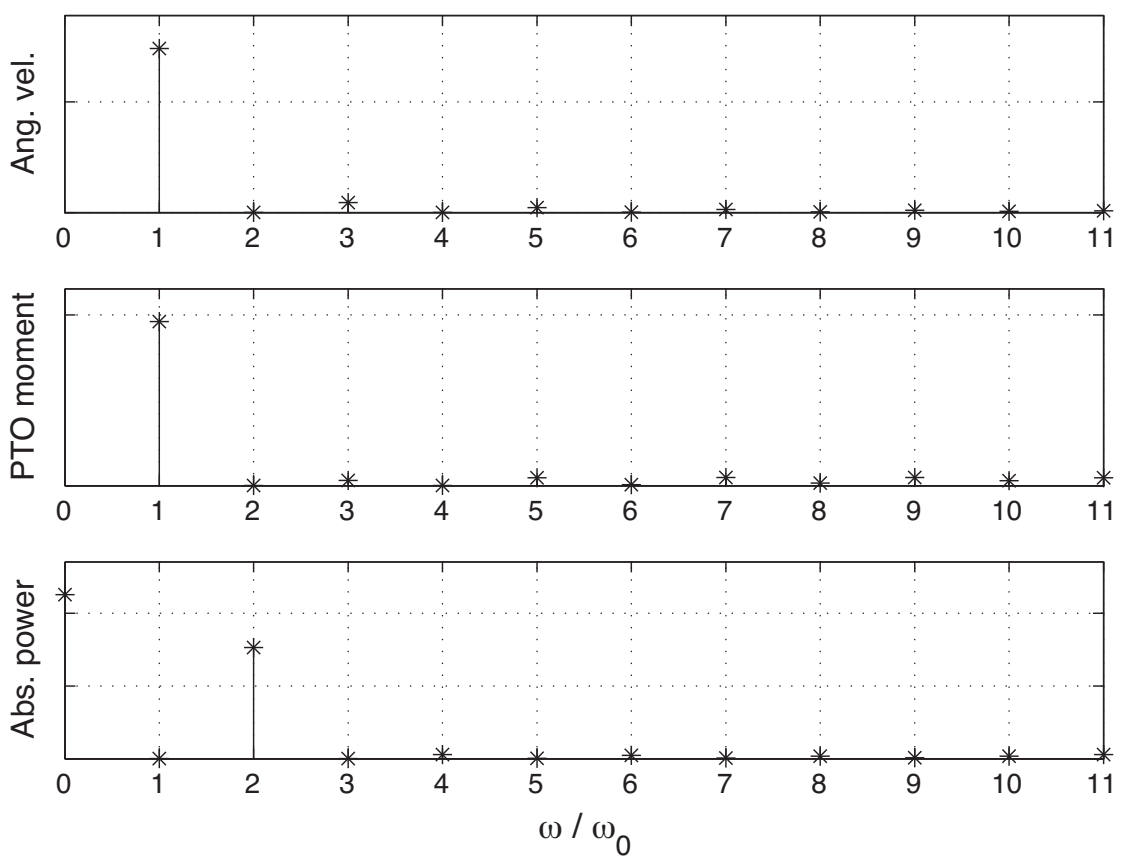

Fig. 4. Spectral components of the velocity, the PTO moment and the absorbed power for $T=10 \mathrm{~s}$ and $\zeta_{a}=2 \mathrm{~m}$.

of the instantaneous power. Using a simple rectangular quadrature over $N_{i}+1$ intervals, we get the following equation,

$J^{N}=d t \sum_{j=0}^{N_{i}} P\left(t_{j}\right)-L\left(r\left(t_{j}\right)\right)\left|P\left(t_{j}\right)\right|$

with, $t_{j}=j \frac{T}{N_{i}+1}, j=0, \ldots, N_{i}$. In order to ensure that the efficiency curve will be well defined for all load values, the PTO power is limited to $P_{\max }$. Thus, the following nonlinear constraint is added to the optimisation problem,

$|P(t)|=\left|\gamma_{p}(t) \dot{\theta}(t)\right| \leq P_{\max }, \forall t \in[0, T]$

The velocity and torque signals are replaced by their approximation in the basis $B=\left\{\phi_{1}, \ldots, \phi_{N}\right\}$ and ensuring inequalities for $N_{p}+1$ points leads to $N_{p}+1$ nonlinear inequality constraints,

$\left|\hat{\mathbf{u}}^{T} \phi^{T}\left(t_{p}\right) \Phi\left(t_{p}\right) \hat{\mathbf{x}}_{2}\right|-P_{\max } \leq 0$

with, $t_{p}=p \frac{T}{N_{p}+1}, p=0, \ldots, N_{p}$. The control problem also includes linear path constraints over the whole optimisation interval, such as angle, velocity and torque limitations, namely,

$\left|x_{1}(t)\right| \leq X_{\max }, \forall t \in[0, T]$

$\left|x_{2}(t)\right| \leq V_{\max }$

$|u(t)| \leq C_{\max }$

$x_{1}, x_{2}$ and $u$ are replaced by their approximation in the basis $B=$ $\left\{\phi_{1}, \ldots, \phi_{N}\right\}$, with the inequalities for $N_{p}+1$ points leading to $6 \times$ $\left(N_{p}+1\right)$ linear inequality constraints,

$\left[\begin{array}{c}\Phi\left(t_{p}\right) \\ -\Phi\left(t_{p}\right)\end{array}\right] \hat{\mathbf{x}}_{1} \leq \mathbf{1}_{2 \times\left(N_{p}+1\right) \times 1} X_{\max }$

$\left[\begin{array}{c}\Phi\left(t_{p}\right) \\ -\Phi\left(t_{p}\right)\end{array}\right] \hat{\mathbf{x}}_{v} \leq \mathbf{1}_{2 \times\left(N_{p}+1\right) \times 1} V_{\max }$
Table 1

Absorbed energy (kW) and computing time (in brackets), as function of the order of the approximation $(N)$ and of the wave period $(T)$ for a wave amplitude of $|\zeta|=2 \mathrm{~m}$.

\begin{tabular}{lllll}
\hline$N$ & $T=4 \mathrm{~s}$ & $T=8 \mathrm{~s}$ & $T=14 \mathrm{~s}$ & $T=20 \mathrm{~s}$ \\
\hline 6 & $563.7(0.16)$ & $1463(0.19)$ & $685.5(0.14)$ & $399.8(0.18)$ \\
10 & $564.5(0.43)$ & $1472(0.31)$ & $687.5(0.32)$ & $401.5(0.37)$ \\
14 & $654.6(0.89)$ & $1473(0.64)$ & $687.7(0.58)$ & $401.6(0.56)$ \\
18 & & $1473(1.17)$ & $687.7(0.98)$ & $401.6(0.79)$ \\
\hline
\end{tabular}

$\left[\begin{array}{c}\Phi\left(t_{p}\right) \\ -\Phi\left(t_{p}\right)\end{array}\right] \hat{\mathbf{u}} \leq \mathbf{1}_{2 \times\left(N_{p}+1\right) \times 1} F_{\max }$

The equation of motion and the derivative relation between position and velocity still have to be satisfied for all the collocation nodes $t_{j}$, defined in Eq. (29), wich constitute $2 N$ equality constraints needed to guarantee proper dynamical behaviour of the WEC. Two types of dynamical model are considered in this study, the first one, derived directly from the standard linear Cummin's equation and a second one, taking into account a quadratic damping term, which is nonlinear. Finally, the first $N$ equality constraints are given by the relation between velocity and position,

$D_{\phi} \hat{\mathbf{x}}_{1}-\hat{\mathbf{x}}_{2}=0$

and the last $N$ equality constraints come from the linear equation of motion expressed at each of the collocation points,

$I_{y} \Phi_{j} \mathrm{D}_{\phi} \hat{\boldsymbol{x}}_{2}+\Phi_{j} \mathrm{G} \hat{\boldsymbol{x}}_{2}+S_{h} \Phi_{j} \hat{\boldsymbol{x}}_{1}-\Phi_{j} \hat{\boldsymbol{u}}-\gamma_{e}\left(t_{j}\right)=0$

or, using the nonlinear equation of motion, include the nonlinear damping terms, so that

$$
\begin{aligned}
& I_{y} \Phi_{j} \mathrm{D}_{\phi} \hat{\boldsymbol{x}}_{2}+B_{v_{1}} \Phi_{j} \hat{\boldsymbol{x}}_{2}+B_{v_{2}} \Phi_{k} \hat{\boldsymbol{x}}_{2}\left|\Phi_{j} \hat{\boldsymbol{x}}_{2}\right|+\Phi_{j} \mathrm{G} \hat{\boldsymbol{x}}_{2}+S_{h} \Phi_{j} \hat{\boldsymbol{x}}_{1} \\
& \quad-\Phi_{j} \hat{\boldsymbol{u}}-\gamma_{e}\left(t_{j}\right)=0
\end{aligned}
$$



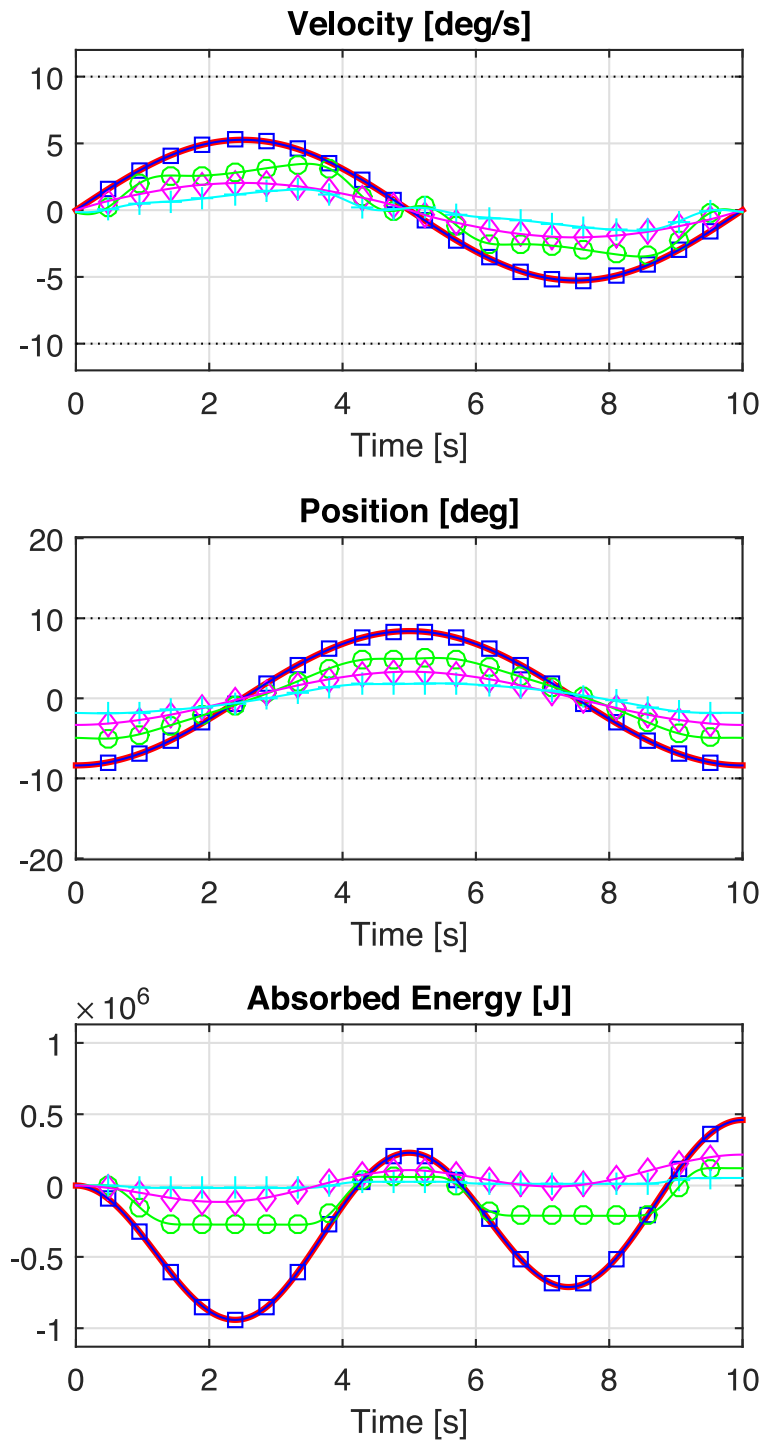
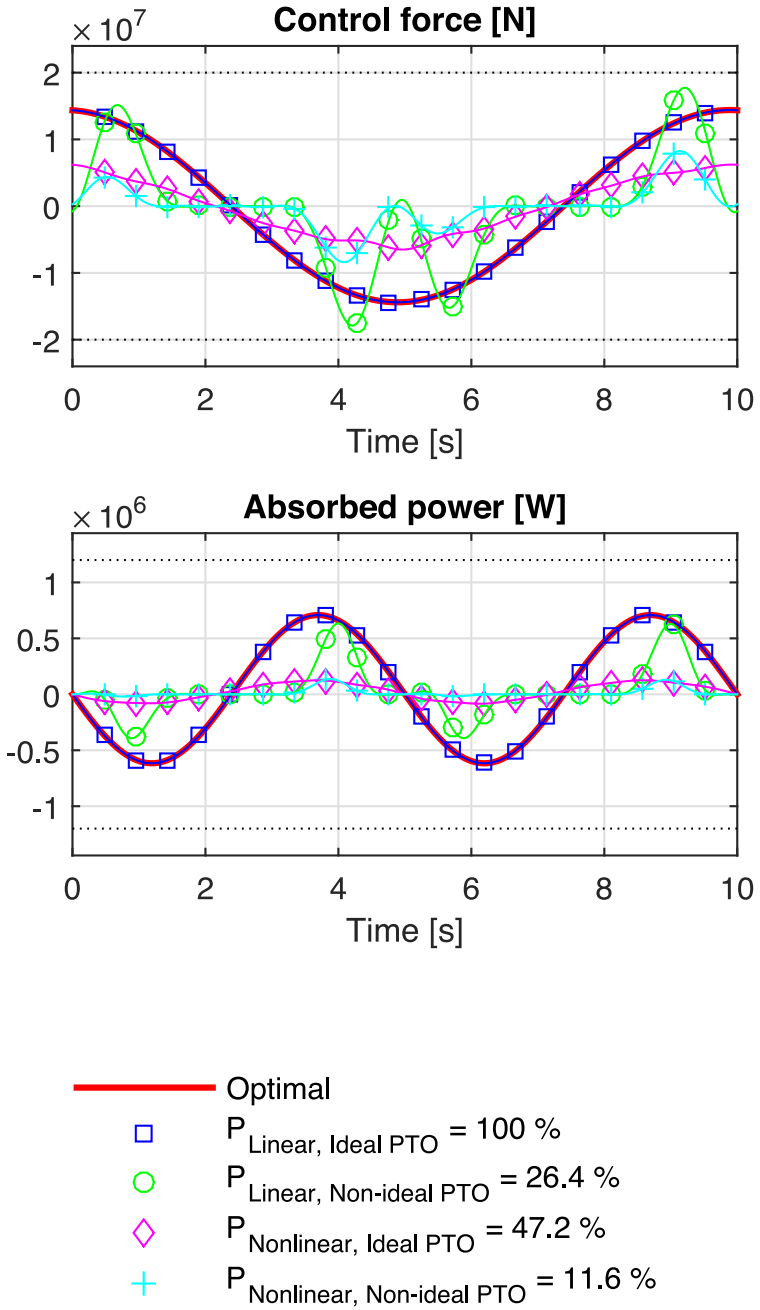

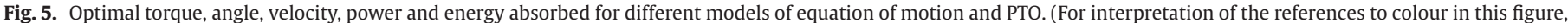
the reader is referred to the web version of this article).

\section{Results}

\subsection{Nonlinear equation of motion}

Evaluations have been carried out in Matlab and the algorithm used for solving the optimisation problem is the Sequential Quadratic Programming algorithm implemented by the fmincon function included in the MATLAB Optimisation Toolbox.

Fig. 3 presents simulation results for an incident wave of amplitude $|\zeta|=2 \mathrm{~m}$ and period $T=10 \mathrm{~s}$, where the state variables $(\theta, \dot{\theta})$ and the control input $\left(\gamma_{p}\right)$ have been approximated using seven frequency components each $(N=14)$. Fig. 3a clearly shows that the controller aims to limit the angular velocity of the device, as the time profile of $\dot{\theta}$ resembles a "flattened" sinusoid and time profile of the angular position seems to approach a motion at constant speed in the time intervals $t \in[1.5,4]$ and $t \in[6,8.5]$. The PTO and the excitation moments are depicted in Fig. 3b while the instantaneous absorbed power is in Fig. 3c. Comparison of Fig. 3a andb also shows that the controller tries to keep the velocity in phase (though phase is badly defined for nonlinear systems) with the excitation force, as happens in the linear case (Falnes, 2002).
Fig. 4 shows the frequency content of the state and control variables, in addition to the absorbed power. The amplitudes of the frequency components decay quickly as the frequency increases, meaning that only a few components are necessary for a good approximation. This is confirmed by looking at Table 1 , where the average absorbed power $\left(P_{u}\right)$, defined as

$P_{u}=\frac{1}{T} J^{N}=\frac{1}{2} \hat{\boldsymbol{u}}^{T} \hat{\boldsymbol{x}}$,

is listed for different values of the expansion order $N$, and for different values of the wave period. The computation times of the optimisation problems, using a laptop computer with a Core i7 processor working at $2.8 \mathrm{GHz}$, are listed in brackets. Based on the results given in Table 1, a value of $N=10$ has been used for the simulations presented in the rest of this paper, as the best trade-off between speed and accuracy. Thus, position, velocity and PTO force are each approximated with a trigonometric polynomial with five frequency components, and the resulting approximate optimal control problem is a nonlinear program with 30 variables and 20 constraints.

In Fig. 6, the average absorbed power is depicted as a function of the wave period and wave amplitude. It is interesting to note that, with the model including a quadratic viscous damping term, the 


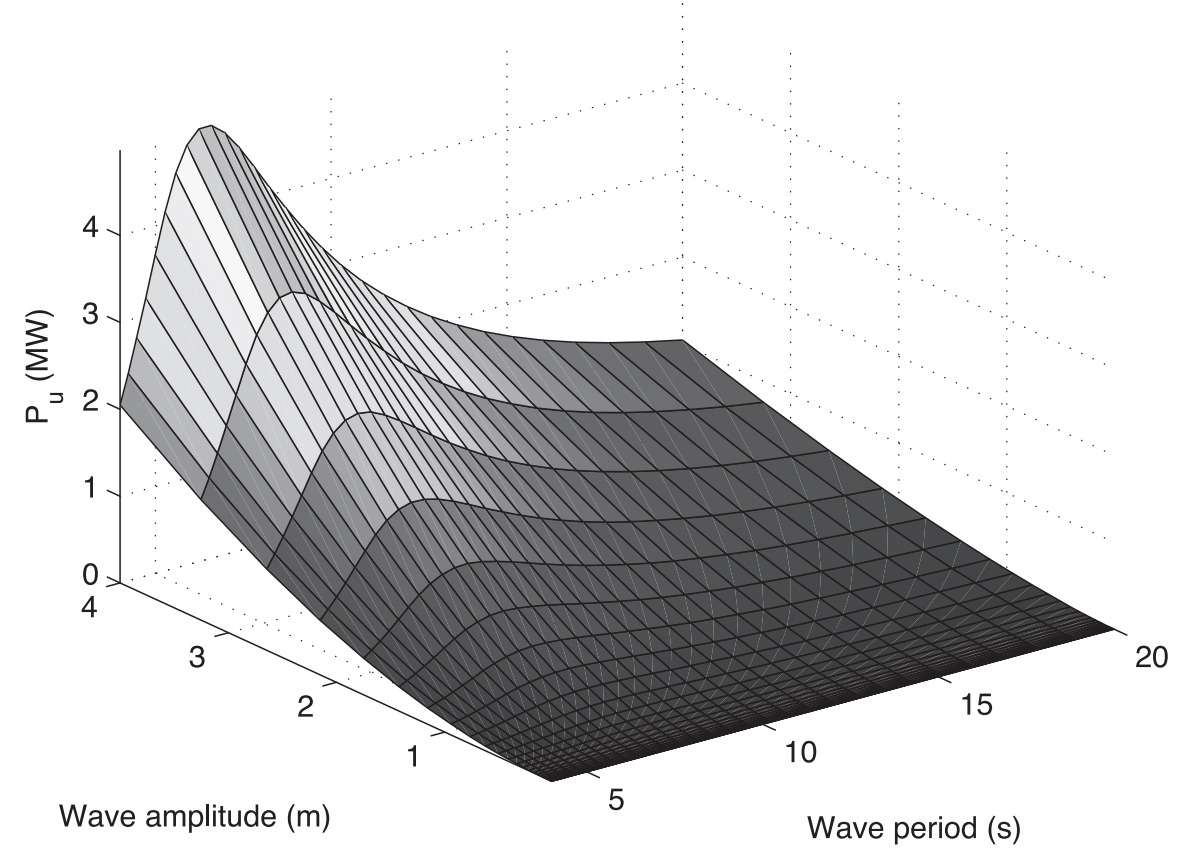

Fig. 6. Average absorbed power $\left(P_{u}\right)$.

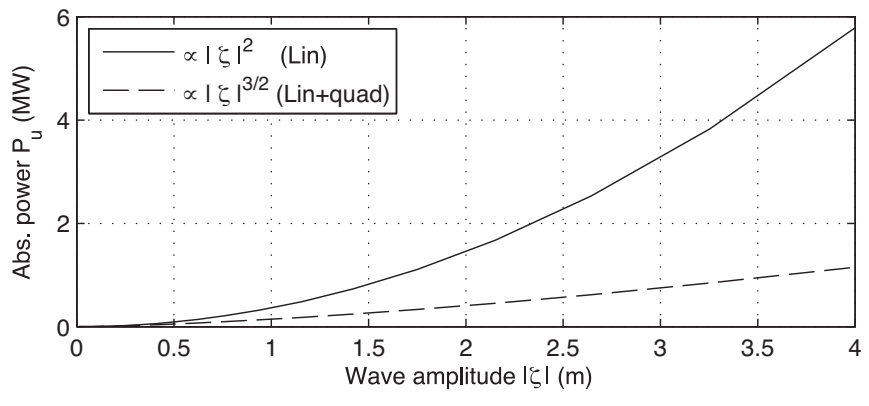

Fig. 7. Absorbed power as function of the wave amplitude for the linear and nonlinear models $(T=20 \mathrm{~s})$. absorbed power increases with $|\zeta|^{\frac{3}{2}}$ rather than $|\zeta|^{2}$, as in the linear case (Falnes, 2002). This fact is highlighted in Fig. 7, where the solid curve is the absorbed power as function of the wave amplitude $|\zeta|$, when only the linear dissipative term is present $\left(B_{v_{2}}=0\right)$; in this case, $P_{u}$ is proportional to the square of the wave amplitude $\left(P_{u} \propto|\zeta|^{2}\right)$. The dashed curve is the absorbed power when the nonlinear term is also included $\left(B_{v_{1}}=0, B_{v_{2}} \neq 0\right)$, and $P_{u}$ increases with the wave amplitude as $P_{u} \propto|\zeta|^{\frac{3}{2}}$.

Of particular interest is Fig. 8, which depicts the ratio of the average absorbed power to the sum of the power radiated and the power dissipated by the linear and quadratic terms, $P_{u} / P_{d}$, where $P_{d}=\frac{1}{T} \int_{0}^{T}\left(B_{v_{1}} \dot{\theta}+B_{v_{2}} \dot{\theta}|\dot{\theta}|+h_{r} * \dot{\theta}\right) \dot{\theta} d t$.

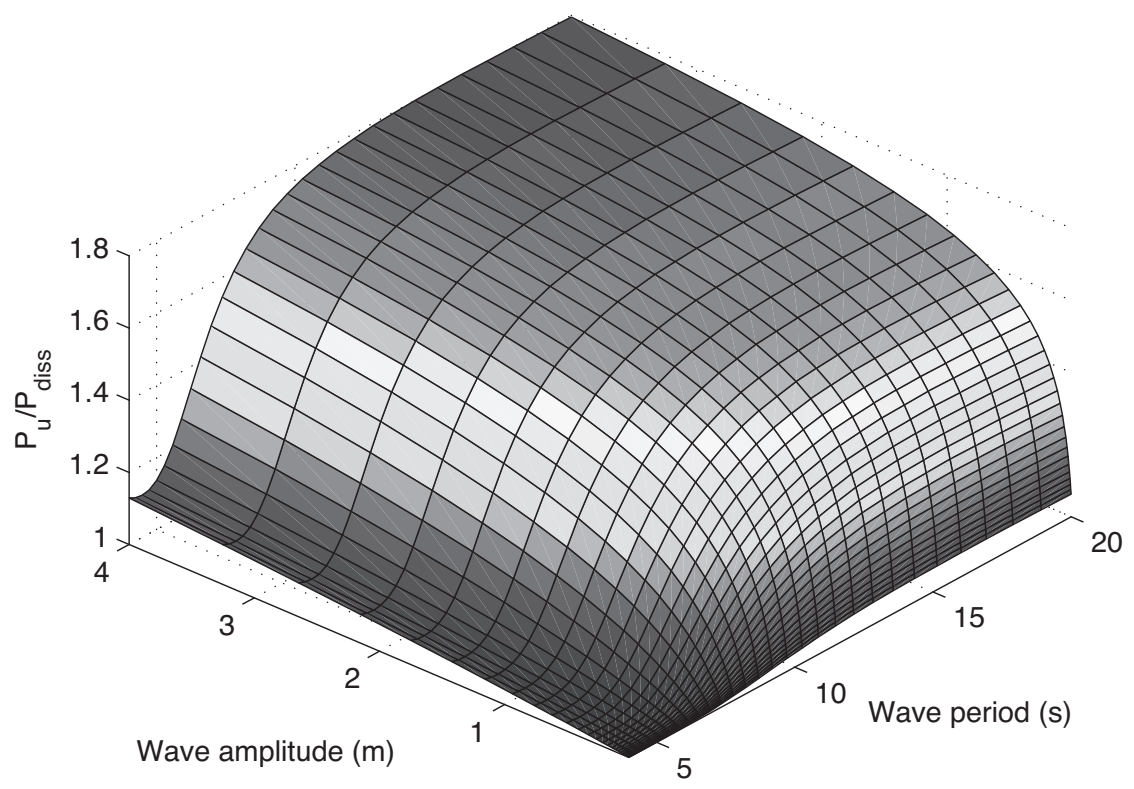

Fig. 8. Ratio of the average absorbed power $\left(P_{u}\right)$ over the dissipated and radiated power. 


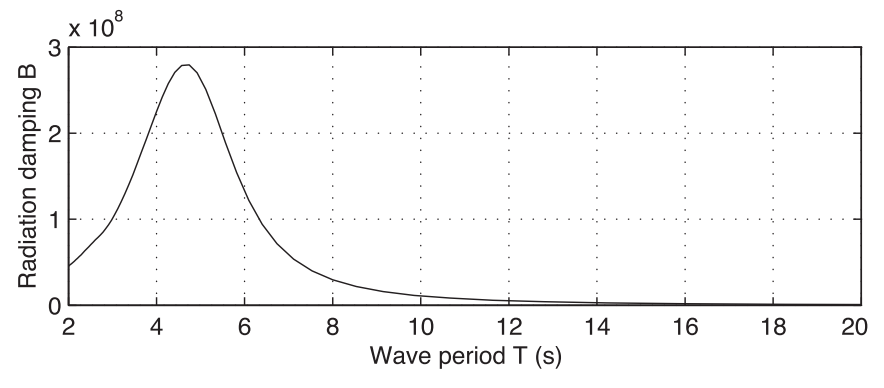

Fig. 9. Radiation damping $B$.

Note that the ratio $P_{u} / P_{d}$ is always greater than one; note that $P_{u} / P_{d}=$ 1 when the model is linear (Falnes, 2002). This fact does not imply that more energy is being absorbed, but only that a larger fraction of the total power flowing through the device is being converted, as the overall absorbed power is smaller because it increases with $|\zeta|^{\frac{3}{2}}$. This result is consistent with linear absorption theory; in fact, when the amplitude of the incident wave is small, the linear dissipative term is dominant with respect to the quadratic term, and the ratio $P_{u} / P_{d} \rightarrow$ 1 , which is what happens in the linear case. The ratio $P_{u} / P_{d}$ becomes close to 1 also when the wave period is close to $T=5 \mathrm{~s}$, for which the linear radiation damping $B$ becomes the dominant term (Fig. 9).

An additional significant difference with the linear case can be observed in Fig. 10, which depicts the ratio of the reactive power over the absorbed power, where the average reactive power is defined as the power that the PTO returns to the oscillating body:

$P_{\text {reac }}=-\frac{1}{T} \int_{0}^{T} \min [P(t), 0] d t$, where $P(t)=\dot{\theta} \gamma_{p}$.

The ratio $P_{\text {reac }} / P_{u}$ is generally small for the range of wave periods and amplitudes considered, when the quadratic term becomes dominant, which is a favourable characteristic when designing a wave energy converter, because PTOs that are unable to return power to the oscillating body are generally less expensive. The consistency with the linear model can also be observed from the results in this figure (Fig. 10) when considering small wave amplitudes, where the amount of reactive power compared to the absorbed power increases considerably. It is well known that, with an optimal linear controller, the amount of reactive power is large when the period of the incident wave is far away from the resonance period (Falnes, 2002).

\subsection{Non-ideal power take-off}

Fig. 5 shows optimal trajectories obtained using a pseudospectral method for a linear and nonlinear equation of motion, and with and without losses in the PTO. Note that the evaluation of the WEC motion is exactly that obtained from the optimal control calculation. Therefore, Fig. 5 shows only results for an identical evaluation and control model. The optimal trajectories, in red in Fig. 5, are obtained from the optimal reactive control, and are used as references to determine the theoretical maximum of the energy absorption rate.

With the linear Cummin's equation and an ideal PTO, in blue with squares in Fig. 5, the trajectory reaches the optimal theoretical solution. Thus, energy absorption is maximal, i.e. equal to $100 \%$. Using the same angle, velocity and torque trajectories, but with a non-ideal PTO, the absorbed energy drops to $-19 \%$. Angle, velocity and torque trajectories with no losses and a linear equation of motion are far from being optimal in the non-ideal case, and are actually losing energy. Optimal trajectories in the ideal case require a significant reactive power in the control process; consequently, the absorbed energy is noticeably penalised by PTO losses.

The second curve in Fig. 5, in green with circles, includes a nonideal PTO in the cost function. The absorbed energy is $26.4 \%$ of the theoretical maximum. By taking into account losses in the control problem formulation, the optimal trajectories drastically improve the quality of the control, and allow the recovery of a quarter of the theoretical maximum, instead of losing energy.

The two last curves in Fig. 5, in magenta with diamonds and cyan with crosses, include a nonlinear damping term in the equation of motion. With an ideal PTO, 52.8\% of the incoming wave energy is lost by viscosity through the nonlinear damping term.

Cyan curves with crosses in Fig. 5, represent a WEC with nonlinear equation of motion and a non-ideal PTO included in the cost function. Resulting position, velocity and control force trajectories allow us to reach $11 \%$ of the theoretical maximum. One can notice that the actual absorbed energy with a non-ideal PTO and nonlinear damping is, for the incident waves considered, approximately ten times smaller than the theoretical optimum. Also, the absorbed power remains almost always positive in the more realistic case, i.e. with a nonlinear

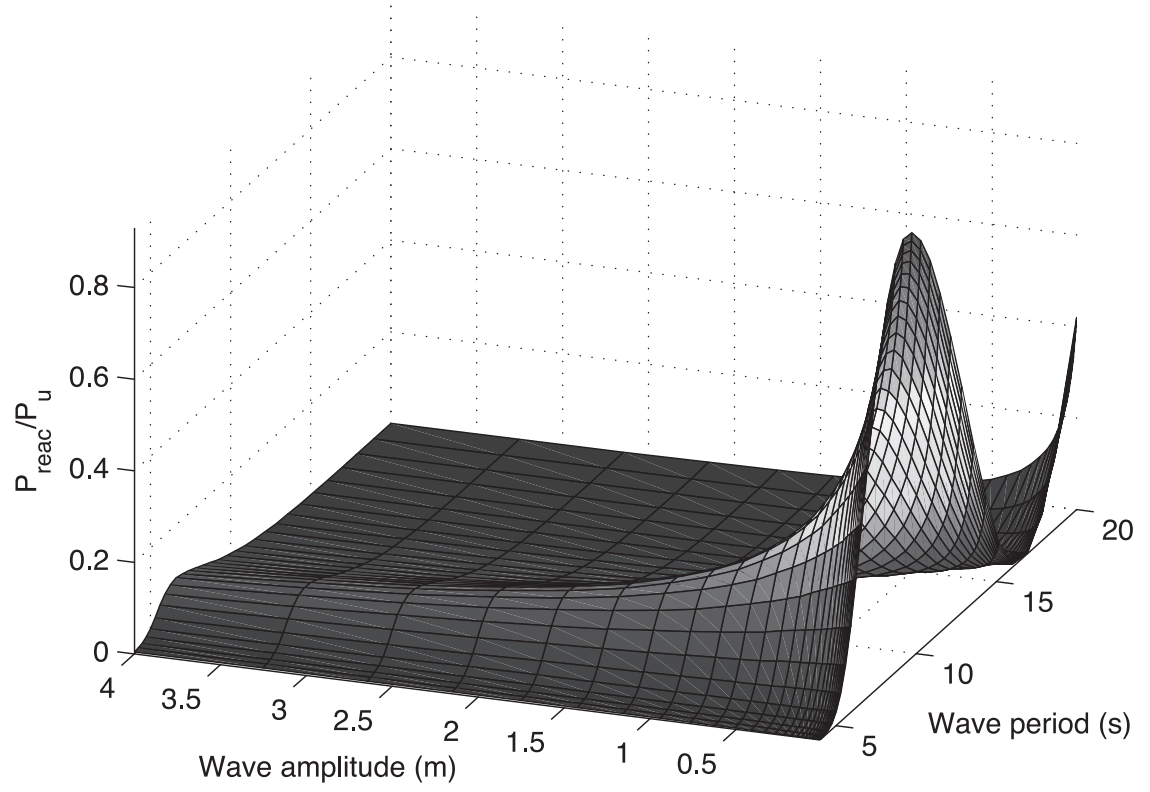

Fig. 10. Ratio of the average reactive power over the average absorbed power. 
dynamical model and non-ideal PTO. In reality, due to losses in the PTO, and from hydrodynamic viscous forces, a reactive actuator might not be essential to follow the optimal trajectory.

\section{Conclusion}

This paper presents a nonlinear WEC control framework using pseudospectral methods, which are known for their beneficial computational and convergence properties, including the benefit that few coefficients are required for obtaining a good approximation, confirmed by the data in Table 1 . The consequence is that the dimension of the nonlinear program and its computing time are small, thus the technique is a good candidate for being implemented in real time applications.

Analysis of results for monochromatic waves at different frequencies and amplitudes show significant differences with linear theory. In particular, the optimal nonlinear controller provides a better ratio between the average absorbed power and dissipated power, when the effect of the nonlinear terms are predominant. This is the case for the hinged flap device which provides the application example. Additionally, the optimal nonlinear control law requires the PTO to return a smaller fraction of reactive power to the oscillating body compared to the optimal linear control law, placing less demands on the PTO system. Furthermore, more cost effective and reliable PTO configurations, such as hydraulic PTOs, which are not well suited for the implementation of linear control laws, due to their strong nonlinearities (Bacelli et al., 2008), can be employed.

The impact of a non-ideal PTO on WEC energy absorption is also investigated, illustrating how crucial it is to take into account PTO losses in the control problem formulation. A tenth of the incoming wave energy can still be recovered under realistic conditions, i.e. with a non-ideal PTO and a nonlinear viscous damping term in the dynamical model of the WEC. Reactive control power requirements become less critical under more realistic conditions, and such conditions lead to an essentially unidirectional energy PTO flux. Passive actuators still remain candidates for control force generation in real sea conditions, reducing cost and maintenance requirements, while ensuring acceptable energy absorption.

Overall, this paper addresses a realistic WEC model containing lossy components, in particular viscous drag and non-ideal PTO efficiency. It is shown, for the case considered, that the observation of these lossy components in the model significantly reduces the captured power fraction, but that this is the best situation that can be achieved. Where the controller model does not reflect the reality of such lossy components, the captured power fraction will reduce further, as demonstrated in Fig. 5. The results, however, are presented for a specific case, where viscous drag is likely to be present, though PTO losses are omnipresent, for all WEC systems. Our recommendation is that realistic models for specific WEC control designs are employed in order to ensure maximum power capture.

\section{Acknowledgement}

The authors would like to thank Dr. Ronan Costello and Thomas Kelly for their support provided on the numerical modelling. This material is based upon works supported by Science Foundation Ireland under Grant no. 13/IA/1886 and Grant no. 12/RC/2302 for the Marine Renewable Ireland (MaREI) centre.

\section{References}

Babarit, A., \& Clément, A. (2006). Optimal latching control of a wave energy device in regular and irregular waves. Applied Ocean Research, 28(2), 77-91.

Bacelli, G., Gilloteaux, J.-C., \& Ringwood, J. (2008). State space model of a hydraulic power take off unit for wave energy conversion employing bondgraphs. In Proceedings 10th world renewable energy congress, WRECX. Glasgow.
Bacelli, G., \& Ringwood, J. V. (2014). Nonlinear optimal wave energy converter control with application to a flap-type device. In Proceedings of the 19th international federation of automatic control, IFAC world congress (pp. 7696-7701). Cape Town, South Africa.

Benson, D. A. (2005). A Gauss pseudospectral transcription for optimal control (Ph.D. thesis). Massachusetts Institute of Technology.

Bhinder, M. A., Babarit, A., Gentaz, L., \& Ferrant, P. (2015). Potential time domain model with viscous correction and CFD analysis of a generic surging floating wave energy converter. International Journal of Marine Energy, 10, 70-96.

Blevins, R. (1992). Applied fluid dynamics handbook. Krieger Publishing Company.

Canuto, C., Hussaini, Y., Quarteroni, A., \& Zang, T. (2006). Spectral methods: fundamentals in single domains. Springer.

Chen, C.-C., Fang, F.-M., Li, Y.-C., Huang, L.-M., \& Chung, C.-Y. (2009). Fluid forces on a square cylinder in oscillating flows with non-zero-mean velocities. International Journal for Numerical Methods in Fluids, 60(1), 79-93.

Cummins, W. (1962). The impulse response function and ship motions. Schiffstechnik, 9, 101-109.

Elnagar, G., Kazemi, M., \& Razzaghi, M. (1995). The pseudospectral legendre method for discretizing optimal control problems. IEEE Transactions on Automatic Control, 40, 1793-1796.

Engja, H., \& Hals, J. (2007). Modelling and simulation of sea wave power conversion systems. In Proceedings of the 7th European wave and tidal energy conference, EWTEC.

Falnes, J. (2002). Ocean waves and oscillating systems: linear interactions including waveenergy extraction. Cambridge University Press.

Folley, M., Whittaker, T., \& van't Hoff, J. (2007). The design of small seabed-mounted bottom-hinged wave energy converters. In Proceedings of the 7th European wave and tidal energy conference, EWTEC. Porto.

Fornberg, B. (1996). A practical guide to pseudospectral methods. Cambridge University Press.

Garg, D., Patterson, M., Hager, W. W., Rao, A. V., Benson, D. A., \& Huntington, G. T. (2010). A unified framework for the numerical solution of optimal control problems using pseudospectral methods. Automatica, 46(11), 1843-1851.

Genest, R., Bonnefoy, F., Clément, A. H., \& Babarit, A. (2014). Effect of non-ideal power take-off on the energy absorption of areactively controlled one degree of freedom wave energy converter. Applied Ocean Research, 48, 236-243.

Gilloteaux, J.-C. (2007). Mouvements de grande amplitude d'un corps flottant en fluide parfait. Application à la récupération de l'énergie des vagues (Ph.D. thesis). Ecole Centrale de Nantes, France.

Hansen, A. H., Pedersen, H. C., \& Andersen, T. O. (2014). Model based feasibility study on bidirectional check valves in wave energy converters. International Journal of Marine Energy, 5, 1-23.

Journé, J., \& Massie, W. (2001). Offshore hydromechanics. Delft University of Technologyy.

Kovaltchouk, T., Multon, B., Ben Ahmed, H., Rongère, F., Aubry, J., \& Glumineau, A. (2013). Influence of control strategy on the global efficiency of a direct wave energy converter with electric power take-off. In Proceedings of the eighth international conference and exhibition on ecological vehicles and renewable energies, EVER.

Merigaud, A., Gilloteaux, J.-C., \& Ringwood, J. (2012). A nonlinear extension for linear boundary element methods in wave energy device modelling. In Proceedings of 31st international conference on ocean, offshore and arctic engineering (OMAE 2012).

Nielsen, S., Zhou, Q., Kramer, M., Basu, B., \& Zhang, Z. (2013). Optimal control of nonlinear wave energy point converters. Ocean Engineering, 72(0), 176-187.

Richter, M., Magana, M., Sawodny, O., \& Brekken, T. (2013). Nonlinear model predictive control of a point absorber wave energy converter. IEEE Transactions on Sustainable Energy, 4(1), 118-126.

Ringwood, J. V., Davidson, J. \& Giorgi, S. (2015). Optimising numerical wave tank tests for the parametric identification of wave energy device models. In Proceedings of the 34th international conference on ocean, offshore and arctic engineering (OMAE 2015). St. John's, Canada.

Ross, I. M., \& Karpenko, M. (2012). A review of pseudospectral optimal control: from theory to flight. Annual Reviews in Control, 36(2), 182-197.

Stengel, R. (1986). Optimal control and estimation. Dover books on advanced mathematics. Dover Publications.

Tedeschi, E., Carraro, M., Molinas, M., \& Mattavelli, P. (2011). Effect of control strategies and power take-off efficiency on the power capture from sea waves. IEEE Transactions on Energy Conversion, 26, 1088-1098.

Tom, N., \& Yeung, R. W. (2013). Non-linear model predictive control applied to a generic ocean-wave energy extractor. In Proceedings of the 32nd international conference on ocean, offshore and arctic engineering (OMAE).

Vlassenbroeck, J., \& Van Dooren, R. (1988). A Chebyshev technique for solving nonlinear optimal control problems. IEEE Transactions on Automatic Control, 33(4), 333-340.

WAMIT (2013). WAMIT inc. USA. URL www.wamit.com.

Zurkinden, A. S., \& Kramer, M. (2012). Numerical time integration methods for a point absorber wave energy converter. In Proceedings of the 27th international workshop on water waves and floating bodies. Copenhagen.

Giorgio Bacelli received the Laurea Magistrale in electronic engineering from the Universita' Politecnica delle Marche, Ancona, Italy, in 2006, and the Ph.D. degree in electronic engineering from the Centre for Ocean Energy Research, Maynooth University, Maynooth, Ireland, in 2014. Currently, he is with Sandia National Laboratories, Albuquerque, NM, USA, working on the design and implementation of control strategies for wave energy converters. 
Romain Genest received a master degree in Hydrodynamics from Ecole Centrale de Nantes, France, and the Ph.D. degree in mechanics from Ecole Centrale de Nantes, France, working on the development and experimental validation of control strategies for wave energy converters. He obtained Bachelor degrees in mechanics and electronics at Ecole Normale Superieure de Rennes, France, and passed the "Agregation" Diploma to teach engineering science in french preparatory school. He is working at Maynooth University, Maynooth, Ireland, as a Post-doctorate researcher in wave energy control.
John V. Ringwood received the Hons. Diploma degree in electrical engineering from Dublin Institute of Technology, Dublin, Ireland, in 1981, and the Ph.D. degree in control of steel mills from Strathclyde University, Glasgow, U.K., in 1985. He is currently Professor of Electronic Engineering at Maynooth University, Maynooth, Ireland. He was the Head of the Electronic Engineering Department, Maynooth University from 2000 to 2006, developing the Department from a green field site, and Dean of Engineering at Maynooth University from 2001-2006. His research interests include time series modelling, wave energy, control of plasma processes, and biomedical engineering. John is a Chartered Engineer and a Fellow of the Institution of Engineers of Ireland. 